\title{
Proposal for teleportation of an atomic state via cavity decay
}

\author{
S. Bose ${ }^{2}$, P. L. Knight ${ }^{1}$, M. B. Plenio ${ }^{1}$ and V. Vedral ${ }^{2}$ \\ ${ }^{1}$ Optics Section, The Blackett Laboratory, Imperial College, London SW' 2BZ, England \\ ${ }^{2}$ Centre for Quantum Computing, Clarendon Laboratory, University of Oxford, Parks Road, Oxford OX1 3PU, England
}

\begin{abstract}
We show how the state of an atom trapped in a cavity can be teleported to a second atom trapped in a distant cavity simply by detecting photon decays from the cavities. This is a rare example of a decay mechanism playing a constructive role in quantum information processing. The scheme is comparatively easy to implement, requiring only the ability to trap a single three level atom in a cavity.
\end{abstract}

Pacs No: 03.67.-a, 03.65.Bz, 42.50.-p, 42.50.Vk

Spontaneous decay is popularly regarded as a coherence loss mechanism in a quantum system. As such, one may not expect such a process to be helpful in quantum information processing [1. Two recent papers [2] 3] tend to dispel this myth by showing how the detection (or the non detection) of decays can be used to entangle the states of distinct atoms. Here, we show that the above approach is not limited to the establishment of entanglement, but can actually be used for genuine quantum information processing such as teleportation 何. In our proposal, the states to be teleported (the "stationary qubits") are atomic states, ideal for the storage of quantum information. Quantum information is physically transferred from place to place via photonic states (the "flying qubits" [5]), which are the best long distance carriers of quantum information. In all experimental implementations of teleportation to date [6], and in some related proposals [9], the stationary qubits have been of "fleeting" optical origin. In earlier proposals of atomic state teleportation [10], the flying qubits have been atomic states and thereby not ideal for long distance teleportation. Our scheme differs from these earlier experiments and proposals in using both the ideal stationary and the ideal flying qubits. It also differs crucially from the much studied quantum communication setup in which a photon directly transfers quantum information from an atom trapped in a cavity to another atom in a distant cavity [5, 11 14. Our scheme does not require a direct carrier of quantum information between distant atoms. Joint detection of photons leaking out of distinct cavities enables disembodied transfer of quantum information from an atom in one of the cavities to an atom in the other. We thus provide a quantum state transfer scheme that avoids the sophisticated task of feeding a photon into a cavity from outside 11, 12].

The setup consists of two optical cavities, each containing a single trapped $\Lambda$ three level atom, as shown in Fig.1. Atoms 1 and 2 are trapped in cavities A and B (supporting cavity modes A and B) respectively.The photons leaking out from both the cavities impinge on the $50-50$ beam splitter $S$ and are detected at the detectors $D_{+}$and $D_{-}$. Initially, we assume unit efficiency detectors (we include finite efficiency later). The cavity A, atom 1, beam splitter $S$ and the detectors $D_{+}$and $D_{-}$belong to Alice. The cavity B with atom 2 belongs to Bob. We require both the cavities to be one sided so that the only leakage of photons occur through the sides of the cavities facing $S$. By following our teleportation protocol, Alice can teleport an unknown state of her atom 1 to the atom 2 held by Bob in three stages.

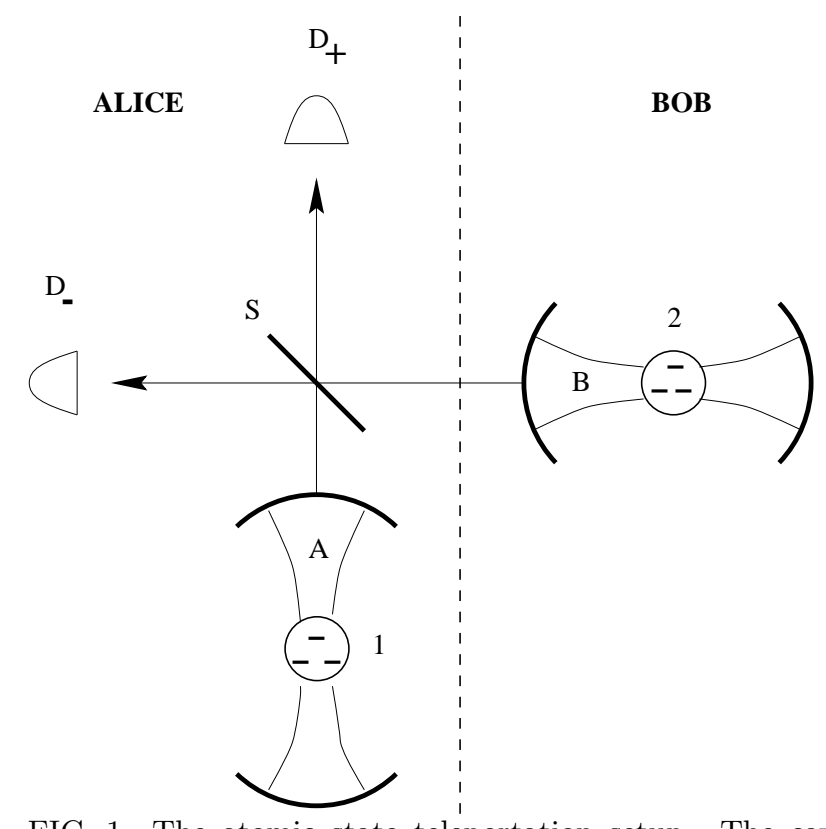

FIG. 1. The atomic state teleportation setup. The cavity A, atom 1 , beam splitter $S$ and the detectors $D_{+}$and $D_{-}$ belong to Alice, while the cavity B and atom 2 belong to Bob.

In the preparation stage, Alice maps her atomic state to her cavity state [15]. At the same time Bob creates a maximally entangled state of his atom and his cavity mode. In the next stage (the detection stage) Alice waits for a finite time for either or both of her detectors to click. If any one of the detectors register a single click during this time period, then the protocol is successful. Otherwise Alice informs Bob about her failure.

This protocol can be related to the standard teleportation protocol [4 by noting that the beam splitter and the detectors constitute a device for measurement of the joint state of the two cavities in the basis $\left\{|0\rangle_{A}|0\rangle_{B},|1\rangle_{A}|1\rangle_{B}, \frac{1}{\sqrt{2}}\left(|0\rangle_{A}|1\rangle_{B}+\right.\right.$ 
$\left.\left.|1\rangle_{A}|0\rangle_{B}\right), \frac{1}{\sqrt{2}}\left(|0\rangle_{A}|1\rangle_{B}-|1\rangle_{A}|0\rangle_{B}\right)\right\}$. Here $\left\{|0\rangle_{A},|1\rangle_{A}\right\}$ and $\left\{|0\rangle_{B},|1\rangle_{B}\right\}$ are photon number states in cavities A and $\mathrm{B}$ respectively. The teleportation is probabilistic, because it is successful only for the pair of Bell state outcomes of the above measurement (later we describe how to convert this to a reliable state transfer protocol). At the end of the detection period, if the protocol has been successful, Alice lets Bob know whether $D_{+}$or $D_{-}$ had clicked. This corresponds to the classical communication part of the standard teleportation protocol [ 4 ]. Dependent on this information Bob applies a local unitary operation to his atom to obtain the teleported state. We call this the post-detection stage.

We now analyse the scheme in detail. As we wish to look at single realizations conditioned on detection (or not) of cavity decays, the ideal unravelling of the system's evolution is through the quantum jump approach [16]. Let the photon decay rate from both the cavities be $\kappa$. While Alice/Bob is applying a Hamiltonian $H$ to her/his atom-cavity system, its evolution subject to no detector click, is governed by the effective Hamiltonian (with $\hbar=1$ ) $H_{\text {eff }}=H-i \kappa c^{\dagger} c$ (where $c^{\dagger}$ and $c$ are the creation and the destruction operators for the cavity mode under consideration). The coherent evolution due to $H_{\text {eff }}$ is interrupted by quantum jumps when there is a click in either the detector $D_{+}$(corresponds to an action of the operator $\left(c_{\mathrm{A}}+c_{\mathrm{B}}\right) / \sqrt{2}$ on the joint state vector of the pair of atom-cavity systems, $c_{\mathrm{A}}$ and $c_{\mathrm{B}}$ being the lowering operators for modes $\mathrm{A}$ and $\mathrm{B}$ respectively) or the detector $D_{-}$(corresponds to an action of the operator $\left(c_{\mathrm{A}}-c_{\mathrm{B}}\right) / \sqrt{2}$ in the same way).

The three level atoms have two ground states $|g\rangle$ and $|e\rangle$ (e.g. Zeeman sub-levels) and an excited state $|r\rangle$ (with a spontaneous decay rate $\gamma$ ) as shown in Fig.2. Alice and Bob use two types of time evolutions of the atom-cavity system as their basic local operations. The first type an adiabatic evolution (shown in Fig.2) which is initiated by switching on a classical laser field which drives the $|e\rangle \rightarrow|r\rangle$ transition with a coupling constant $\Omega$. The $|r\rangle \rightarrow|g\rangle$ transition is driven by the quantized cavity mode of coupling $g$. Both the classical laser field and the cavity modes are assumed to be detuned from their respective transitions by the same amount $\Delta$. As the atom is trapped in a specific position in the cavity, we can assume that the couplings $\Omega$ and $g$ remain constant during the interaction. We choose parameters such that $g \Omega / \Delta^{2} \ll 1$ (the upper level $|r\rangle$ can then be decoupled from the evolution) and $\Delta \gg \gamma$ (the spontaneous decay rate from $|r\rangle$ can be neglected). The Hamiltonian for the evolution of the system under such conditions (and assuming $g=\Omega$ for simplicity), is given by $H^{(1)}=E|e\rangle\langle e|+E| g\rangle\langle g|+E\left(c|e\rangle\left\langle g\left|+c^{\dagger}\right| g\right\rangle\langle e|\right)$ where $E=g \Omega / \Delta[12]$. The other local operation accessible to Alice and Bob is the Zeeman evolution used to give an arbitrary phase shift of the level $|e\rangle$ relative to the level $|g\rangle$. The Hamiltonian for this evolution is $H^{(2)}=\delta E|e\rangle\langle e|$, where $\delta E$ is an energy difference.

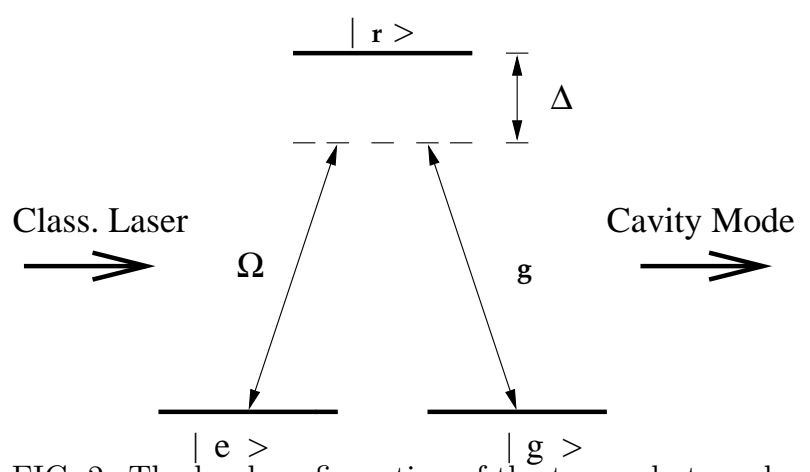

FIG. 2. The level configuration of the trapped atom showing the fields responsible for the adiabatic evolution. The $|e\rangle \rightarrow|r\rangle$ transition being driven by a classical laser field of coupling $\Omega$ and the $|r\rangle \rightarrow|g\rangle$ transition being driven by the quantized cavity mode of coupling $g . \Delta$ is the detuning of both the classical laser field and the quantized field mode from their respective transitions.

Let the unknown state of the atom 1 which Alice wants to teleport be

$$
|\Psi\rangle_{1}^{I}=a|e\rangle_{1}+b|g\rangle_{1}
$$

where the superscript $I$ in $|\Psi\rangle_{1}^{I}$ stands for input and $a$ and $b$ are complex amplitudes. We will assume that the initial state of Alice's cavity is $|0\rangle_{A}$ and the initial state of Bob's atom-cavity system is $|e\rangle_{2}|0\rangle_{B}$. At first, Alice maps the state of atom 1 onto the cavity mode $A$ by switching the Hamiltonian $H^{(1)}$ on for a period of time $t_{I}$ given by $\tan \frac{\Omega_{\kappa} t_{I}}{2}=-\frac{\Omega_{\kappa}}{\kappa}$ where $\Omega_{\kappa}=\sqrt{4 E^{2}-\kappa^{2}}$. Subject to no decay being recorded in the detectors, the cavity state is given by

$$
|\Psi\rangle_{A}^{I}=\frac{1}{\sqrt{|a|^{2} \alpha^{2}+|b|^{2}}}\left(a \alpha|1\rangle_{A}+b|0\rangle_{A}\right),
$$

where $\alpha=\left(\frac{e^{-\frac{\kappa}{2} t_{I}}}{\Omega_{\kappa}} 2 E \sin \frac{\Omega_{\kappa} t_{I}}{2}\right)$. The probability that no photon decay takes place during this evolution is given by $P_{N D}(A)=\left(|a|^{2} \alpha+|b|^{2}\right)$. Meanwhile, Bob also switches on the Hamiltonian $H^{(1)}$ in his cavity for a shorter length of time $t_{E}$ given by $\tan \frac{\Omega_{\kappa} t_{E}}{2}=-\frac{\Omega_{\kappa}}{2 E-\kappa}$. His atom-cavity system thus evolves to the entangled state

$$
|\Psi\rangle_{2, B}^{E}=\frac{1}{\sqrt{2}}\left(|e\rangle_{2}|0\rangle_{B}+i|g\rangle_{2}|1\rangle_{B}\right)
$$

The probability that no photon decay takes place during this evolution is given by $P_{N D}(B)=|\beta|^{2}$ where $\beta=\frac{e^{-\frac{\kappa}{2} t_{E}}}{\Omega_{\kappa}} 2 \sqrt{2} E \sin \frac{\Omega_{\kappa} t_{E}}{2}$. For simplicity, we assume that Alice and Bob synchronize their actions such that the preparation of the states $|\Psi\rangle_{A}^{I}$ and $|\Psi\rangle_{2, B}^{E}$ terminate at the same instant of time. This concludes the preparation stage of the protocol. The probability that this stage is a success is the probability that no photon decays from either cavity during the preparation. This is given by $P_{\text {suc }}($ prep $)=P_{N D}(A) P_{N D}(B)$. We will choose $\Omega_{\kappa}>>\kappa$ which makes $P_{\text {suc }}$ (prep) $\sim 1$. 
Now comes the detection stage, in which Alice simply waits for any one of the detectors $D_{+}$or $D_{-}$to click. She waits for a finite detection time denoted by $t_{D}$. Alice and Bob reject the cases in which Alice does not register any click or registers two clicks. The joint state of Alice's and Bob's system at the begining of the detection stage is

$$
|\Phi(0)\rangle=|\Psi\rangle_{A}^{I} \otimes|\Psi\rangle_{2, B}^{E}
$$

Assume Alice registers a single click at a time $t_{j} \leq t_{D}$. The joint state of Alice's and Bob's system evolves as $|\Phi(t)\rangle_{A, 2, B}=|\Psi(t)\rangle_{A}^{I} \otimes|\Psi(t)\rangle_{2, B}^{E}$ [16], where $|\Psi(t)\rangle_{A}^{I}=$ $\left(a \alpha e^{-\kappa t}|1\rangle_{A}+b|0\rangle_{A}\right) / \sqrt{|a \alpha|^{2} e^{-2 \kappa t}+|b|^{2}}$ and $|\Psi(t)\rangle_{2, B}^{E}=$ $\left(|e\rangle_{2}|0\rangle_{B}+i e^{-\kappa t}|g\rangle_{2}|1\rangle_{B}\right) / \sqrt{1+e^{-2 \kappa t}}$. The registering of a click at one of the detectors corresponds to the action of the jump operators $\left(c_{\mathrm{A}} \pm c_{\mathrm{B}}\right) / \sqrt{2}$ on the state $\left|\Phi\left(t_{j}\right)\right\rangle_{A, 2, B}$. Then the resultant joint state of Alice's and Bob's system becomes

$$
\begin{aligned}
\left|\Phi\left(t_{j}\right)\right\rangle_{A, 2, B}^{J \pm} & =\frac{1}{\sqrt{P_{N D}(A)+2|a|^{2} \alpha^{2} e^{-2 \kappa t_{j}}}}\left\{\left(a \alpha|e\rangle_{2}\right.\right. \\
& \left. \pm i b|g\rangle_{2}\right) \otimes|0\rangle_{A}|0\rangle_{B} \\
& \left.+e^{-\kappa t_{j}} a \alpha|g\rangle_{2} \otimes\left(|1\rangle_{A}|0\rangle_{B} \pm|0\rangle_{A}|1\rangle_{B}\right)\right\}
\end{aligned}
$$

$\left|\Phi\left(t_{j}\right)\right\rangle_{A, 2, B}^{J \pm}$ corresponds to the click being registered in $D_{ \pm}$and the superscript $J$ stands for jump. At the end of a successful detection stage the joint state of the cavities $\mathrm{A}, \mathrm{B}$ and atom 2 will be $\left|\Phi\left(t_{D}\right)\right\rangle_{A, 2, B}^{J \pm}$. In the post detection stage, Bob uses $H^{(2)}$ to give $|g\rangle_{2}$ an extra phase shift with respect to $|e\rangle_{2}$. This phase shift is $-i$ if $D_{+}$ had clicked and $i$ if $D_{-}$had clicked. This concludes the entire protocol.

We now proceed to estimate the fidelity of the teleported state generated at Bob's end with respect to Alice's input state $|\Psi\rangle_{1}^{I}$. First we must note that though the field continues to decay even after the protocol is over (i.e Alice has ceased to keep track of detector clicks), the reduced density matrix of atom 2 remains unchanged, as this atom no longer interacts with the cavity field. Thus the average density matrix of Bob's atom generated due to our teleportation procedure is given by $\rho_{2}^{T e l}=\left\{P_{N D}(A)|\Psi\rangle_{2}\left\langle\left.\Psi\right|_{2}+\right.\right.$ $2|a|^{2} \alpha^{2} e^{-2 \kappa t_{D}}|g\rangle_{2}\left\langle\left. g\right|_{2}\right\} /\left\{P_{N D}(A)+2|a|^{2} \alpha^{2} e^{-2 \kappa t_{D}}\right\}$, where $|\Psi\rangle_{2}=\left(a \alpha|e\rangle_{2}+b|g\rangle_{2}\right) / \sqrt{|a|^{2} \alpha^{2}+|b|^{2}}$. The fidelity of this state with respect to the input state is $F\left(t_{D}, a, b\right)=\left\{P_{N D}(A)\left(|a|^{2} \alpha+|b|^{2}\right)+\right.$ $\left.2|a|^{2} \alpha^{2} e^{-2 \kappa t_{D}}|b|^{2}\right\} /\left\{P_{N D}(A)+2|a|^{2} \alpha^{2} e^{-2 \kappa t_{D}}\right\}$. We see that apart from the system parameters $\kappa$ and $\Omega_{\kappa}$, the fidelity of the generated state also depends on the detection time $t_{D}$ and the modulus of the amplitudes $a$ and $b$ of the initial state. It is a teleportation protocol with a state dependent fidelity. The fidelity does not depend on $P_{N D}(B)$ because the initial state $|\Psi\rangle_{2, B}^{E}$ prepared by Bob is independent of the decay rate of his cavity.

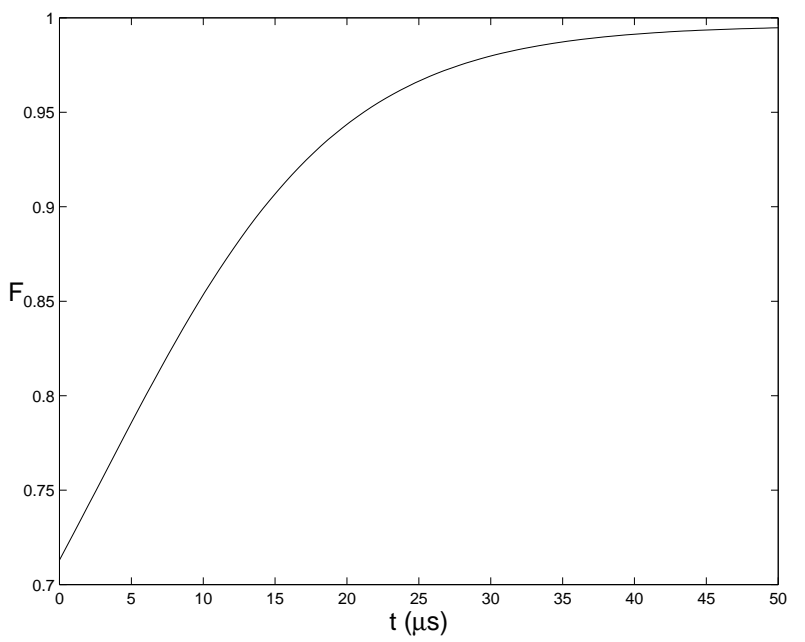

FIG. 3. The improvement of average teleportation fidelity with the length of the detection stage. The parameter regime is $(g: \Omega: \kappa: \gamma: \Delta) / 2 \pi=(10: 10: 0.01: 1: 100) \mathrm{MHz}$

We plot the variation of the average fidelity of teleportation over all possible input states as a function of the detection time $t_{D}$ in Fig. 3. We see that the fidelity increases with increasing detection time. This happens because increasing the detection time decreases the proportion of $|g\rangle_{2}\left\langle\left. g\right|_{2}\right.$ in the teleported state $\rho_{2}^{T e l}$ and brings it closer to the initial state $|\Psi\rangle_{1}^{I}$ of Alice's atom. The parameter regime used for Fig. $3\{(g: \Omega: \kappa: \gamma: \Delta) / 2 \pi=$ $(10: 10: 0.01: 1: 100) \mathrm{MHz}\}$, is carefully chosen to satisfy all our constraints $\left(g \Omega / \Delta^{2} \ll 1, \Delta>>\gamma, \Omega_{\kappa}>>\kappa\right)$. This regime could be approached, for example, by increasing the cavity finesse of Ref. 117] by an order of magnitude and increasing the length of that cavity to about a millimeter while keeping the beam waist and other parameters constant. As evident from Fig.3, the average fidelity exceeds 0.99 for a detection time of about half the cavity life time.

The total probability of success of the protocol is also state dependent and given by $P_{\text {suc }}=P_{\text {suc }}($ prep $) \times$ $P_{1 D}\left(0, t_{D}\right)=\left(P_{N D}(A)+2|a|^{2} \alpha^{2} e^{-2 \kappa t_{D}}\right) P_{N D}(B)(1-$ $\left.e^{-2 \kappa t_{D}}\right) / 2$, where $P_{1 D}\left(0, t_{D}\right)$ is the probability of a single decay during the detection period. In the parameter domain under consideration, for $t_{D}=50 \mu \mathrm{s}$, we find that the average of the probability of success over all input states is about 0.49 . This is a little lower than the ideal success probability of 0.5 (for Alice registering any of the pair of Bell state outcomes) because the preparation stage has an extremely small, but finite, chance of failure.

Let, in a real experiment, the total efficiency of photon detection (including all detector and other unwanted losses) be $\eta$. In the detection stage, Alice will be able to detect only a fraction $\eta$ of all her successful protocols. On the other hand, during this stage, she will erroneously regard a fraction $2 \eta(1-\eta)$ of the cases with two decays as successful cases. Then the probability of a successful protocol changes to $P_{\text {suc }}(\eta)=\eta P_{1 D}\left(0, t_{D}\right)+2 \eta(1-\eta)(1-$ $\left.P_{N D}\left(0, t_{D}\right)-P_{1 D}\left(0, t_{D}\right)\right)$, and the fidelity of the fidelity of the teleported state would be $\left\{\eta P_{1 D}\left(0, t_{D}\right) F\left(t_{D}, a, b\right)+\right.$ $\left.2 \eta(1-\eta)\left(1-P_{N D}\left(0, t_{D}\right)-P_{1 D}\left(0, t_{D}\right)\right)|b|^{2}\right\} / P_{\text {suc }}(\eta)$, where 
$P_{N D}\left(0, t_{D}\right)$ is the probability of no decay during the detection period. In the parameter domain under consideration, and for $\eta$ not lower than 0.1 , we can neglect the effect of undetected photon losses during the preparation stage on the fidelity. For a $\eta$ of 0.6 and detection times large compared to the cavity decay time, the fidelity of the state at Bob's end becomes $\sim 0.81$.

The main practical role of teleportation is to act as a device to link up distant quantum processors with entanglement. To set up entanglement between their atoms, Alice and Bob must both prepare their respective atom-cavity systems in the state $(|e\rangle|0\rangle+i|g\rangle|1\rangle) / \sqrt{2}$ during the preparation stage. Entanglement between the atoms is established if there is a single click during the detection period. The resultant entangled state is $\left|\Psi_{12}\right\rangle=\left\{\eta\left(1-e^{-4 \kappa t}\right) / 4\right\}\left|\psi^{ \pm}\right\rangle\left\langle\psi^{ \pm}\right|+\{\eta(1-$ $\left.\eta)\left(1+e^{-4 \kappa t}-2 e^{-2 \kappa t}\right) / 2\right\}|g\rangle_{1}|g\rangle_{2}\left\langle\left. g\right|_{1}\left\langle\left. g\right|_{2}\right.\right.$, where $\left.\mid \psi^{ \pm}\right\rangle=$ $|e\rangle_{1}|g\rangle_{2} \pm|g\rangle_{1}|e\rangle_{2}$. The relative entropy of entanglement of this state can be calculated [18] and for $t_{D}$ large compared to the cavity decay time and a reasonable $\eta$ of 0.6 it is about 0.16 , while for a high $\eta$ of 0.9 , it is about 0.48 (note that $\left|\Psi_{12}\right\rangle$ is entangled for arbitrary $\eta$ ). From the viewpoint of setting up of entanglement, our scheme is rather close to the scheme described by Cabrillo et al. [2]. But the efficiency of success can be much higher (nearing 0.5).

The above probabilistic teleportation protocol can be modified to teleportation with insurance, so that in the cases when the protocol is unsuccessful, the original state of Alice's atom 1 is not destroyed, but mapped onto another reserve atom $r$ trapped in Alice's cavity. To accomplish this, Alice has to follow the local redundant encoding of Ref. 13 and codes her initial state $|\Psi\rangle_{1}^{I}$ as $a\left(|e\rangle_{1}|g\rangle_{r}+|g\rangle_{1}|e\rangle_{r}\right)+b\left(|g\rangle_{1}|g\rangle_{r}+|e\rangle_{1}|e\rangle_{r}\right)$. After this, she just follows the same protocol as before. But in cases when the protocol is unsuccessful, she is left with either the state $a|g\rangle_{r}+b|e\rangle_{r}$ or a state that can be converted to $a|g\rangle_{r}+b|e\rangle_{r}$ by a known unitary transformation. She can now exchange the roles of atom 1 and atom $r$ and try to teleport the state $|\Psi\rangle_{1}^{I}$ again. She can repeat this procedure until teleportation is successful (Of course, this holds true perfectly only when $\eta=1$ ).

To conclude, we have presented a simple scheme for atomic state teleportation, which could be implemented by trapping single three level atoms in a cavity. Moreover, by adding one more atom to Alice's cavity, it can be converted to a reliable state transfer protocol. This state transfer protocol can be viewed as an alternative to designer laser pulses for transferring (Refs. 11, 12]) quantum information into a cavity from outside. This state transfer should work for distances of the order of the absorption length scales of a fibre. The model independent portions of the analysis of communication through a noisy quantum channel [13, 14, 19] should carry over to this decay-induced scenario of state transfer. The scheme described here is also a rare example of a quantum decay playing a constructive role in quantum information processing.

We thank S. L. Braunstein, W. Lange, E. Paspalakis and S. J. van Enk for valuable discussions. Part of this work was carried out during the Complexity, Computation and Physics of Information Programme of the Isaac Newton Institute, and the European Science Foundation QIT-Programme on Quantum Information Theory. This work was supported in part by the Inlaks Foundation, the UK Engineering and Physical Sciences Research Council, the European Union, the Leverhume Trust, Elsag-Bailey and Hewlett-Packard.

[1] M. B. Plenio and P. L. Knight, Phys. Rev. A 53, 2986 (1996); Proc. Roy. Soc. A 453, 2017 (1997).

[2] C. Cabrillo et al., Phys. Rev. A 59, 1025 (1999).

[3] M. B. Plenio et al., Phys. Rev. A 59, 2468 (1999); See also, G. J. Yang et al., Phys. Rev. A 59, 4012 (1999).

[4] C. H. Bennett et al., Phys. Rev. Lett. 70, 1895 (1993).

[5] K. M. Gheri et al., Fortsch. Phys. 46, 401 (1998).

[6] D. Bouwmeester et al., Nature (London) 390, 575 (1997).

[7] D. Boschi et al., Phys. Rev. Lett. 80, 1121 (1998).

[8] A. Furusawa et al., Science 282, 706 (1998); S. L. Braunstein and H. J. Kimble, Phys. Rev. Lett. 80, 869 (1998).

[9] G. J. Milburn and S. L. Braunstein, LANL e-print quantph/9812018; T. C. Ralph and P. K. Lam, Phys. Rev. Lett. 81, 5668 (1998); C. J. Villas-Bôas et al., LANL e-print quant-ph/9904028; M. S. Zubairy, Phys. Rev. A 58, 4368 (1998).

[10] L. Davidovich et al., Phys. Rev. A 50, R895 (1994); J. I. Cirac and A. S. Parkins, Phys. Rev. A 50, R4441 (1994); M. H. Y. Moussa, Phys. Rev. A 55, R3287 (1997); S.-B. Zheng and G.-C. Guo, Phys. Lett. A 232, 171 (1997).

[11] J. I. Cirac et al., Phys. Rev. Lett. 78, 3221 (1997); S. J. van Enk et al., Phys. Rev. A 59, 2659 (1999).

[12] T. Pellizzari, Phys. Rev. Lett. 79, 5242 (1997);

[13] S. J. van Enk et al., Phys. Rev. Lett. 78, 4293 (1997).

[14] S. J. van Enk et al., Phys. Rev. Lett. 79, 5178 (1997); Science 279, 205 (1998); A. Sørensen and K. Mølmer, Phys. Rev. A 58, 2745 (1998).

[15] A. S. Parkins et al., Phys. Rev. A 51, 1578 (1995).

[16] M. B. Plenio and P. L. Knight, Rev. Mod. Phys. 70, 101 (1998).

[17] C. J. Hood et al., Phys. Rev. Lett. 80, 4157 (1998); See also, J. Ye et al. LANL e-print quant-ph/9908007.

[18] V. Vedral and M. B. Plenio, Phys. Rev. A 57, 1619 (1998); V. Vedral et al., Phys. Rev. Lett. 78, 2275 (1997).

[19] H.-J. Briegel et al., Phys. Rev. Lett. 81, 5932 (1998). 